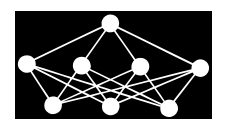

\title{
NEURAL-NETWORK-BASED GENETIC ALGORITHM FOR OPTIMAL KITCHEN FAUCET STYLES
}

\author{
F.B. Ozsoydan ${ }^{*}$ C.M. Kandemir $†$ E.A. Demirtas ${ }^{\ddagger}$
}

\begin{abstract}
Artificial neural networks (ANNs) are the models of choice in many data classification tasks. In this study, ANN classification models were used to explore user perceptions about kitchen faucet styles and investigate the relations between the overall preferences and kansei word scores of users. The scores given by consumers were obtained via a two-stage questionnaire mentioned in a previous study by the authors. Through the questionnaire, consumers were asked to give scores after examining three-dimensional (3-D) drawings of new product samples created with the help of industrial product designers. Because it was neither practical nor necessary to develop a prototype or a picture of each of the alternative designs, a fractional factorial experimental design similar to Taguchi's L-16 orthogonal array was used. After completing this preparatory work to develop ANNs and obtain the necessary related data, an analysis of variance (ANOVA) was performed to identify the critical factors that affect the accuracy of the ANN model to be used and determine the best factor levels for the ANN model. A genetic algorithm (GA) was then integrated with the ANN model found to be the best and implemented to determine the optimal levels of the design parameters related to product appearance. Lastly, the product categories were classified as unfavorable or favorable, and three products were derived for each category. In comparison with the previously published papers of the authors, the GA integrated with the ANN model was found to be an effective tool for revealing user perceptions in new product development. In regard to the findings of the present work, it can be said that, this technique can be used as an alternative of several complex analytical approaches, in order to explore users' perceptions.
\end{abstract}

Key words: kitchen faucets, styling, artificial neural networks, genetic algorithms

Received: December 16, 2013

DOI: $10.14311 / \mathrm{NNW} .2015 .25 .020$

Revised and accepted: November 25, 2014

*Fehmi Burcin Ozsoydan - Corresponding author, Dokuz Eylul University, Industrial Engineering Department, 35160, Izmir, Turkey, Tel: +90 23230176 30, E-mail: burcin.ozsoydan@deu.edu.tr

${ }^{\dagger}$ Celal Murat Kandemir, Eskisehir Osmangazi University, Teaching Computer Education and Instructional Technology Department, 26480, Eskisehir, Turkey, Tel: +90 22223937 50, E-mail: kandemir@ogu.edu.tr

${ }_{\ddagger}$ Ezgi Aktar Demirtas, Eskisehir Osmangazi University, Industrial Engineering Department, 26480, Eskisehir, Turkey, Tel: +90 22223937 50, E-mail: eaktar@ogu.edu.tr 


\section{Introduction}

In today's competitive environment, companies need to understand their customer expectations as well as possible and develop and improve product designs to increase customer satisfaction. Product development approaches such as quality function deployment are helpful in the design of user-oriented products in that they focus attention on aspects of the product such as performance, reliability, durability and cost. While expectations related to product performance have been important in current studies, an increasing number of studies have focused on consumers' emotional expectations of a product or service [10, 24, 33, 36]. In addition, visual product attributes, such as the shape of a handle or the body of a kitchen faucet, must be considered during the design process because these attributes have a substantial influence on consumer purchase decisions [3, 37]. Although some methods have been created to account for emotional responses to consumer products in the design process, these methods are limited by their dependence on subjective scales such as semantics [5, 20, 25]. To gain inspiration for new designs, some designers commonly use data on previously developed designs and rely on their design experience [34]; however, designers and consumers may differ in their perceptions of a product $[2,15]$. Kansei engineering, developed by Nagamachi at Hiroshima University, is used to measure users' feelings and perceptions about a product and consider those feelings and perceptions in the product design process $[25,26]$. A similar approach was researched by Lai et al. [19] to quantify visceral judgments. In that study, the feeling quality of vehicle profiles was assessed. This approach is similar to kansei engineering. The only difference is that the emotive qualities of a product are assessed in kansei engineering.

It is possible to classify kansei engineering (KE) applications described in the literature into two groups. Studies in the first group focus on the determination of user feelings (described by kansei words) toward a product or a product group and the determination of product styles or images. Studies in the second group focus on the determination of kansei words that affect users' overall preferences and the relations between kansei words and design parameter levels. Researchers have used linear regression and correlation analysis to identify kansei words that are related to users' overall preferences. Researchers have also used statistical methods such as Hayashi's quantification theory and conjoint analysis to characterize the relations between overall preferences or words and design parameter levels [6]. Several researchers have used artificial neural networks (ANNs) [13, 21], fuzzy ANNs [14] gray relational analysis combined with ANNs [20] and genetic algorithms (GAs) $[12,16,17,31]$ for the same purpose. A number of other researchers have compared the performance of traditional statistical methods, such as logistic regression, to the performance of ANNs in solving diverse real-life problems [29]. Dreiseitl and Machado [7] reviewed 72 papers that compared the classification performance of ANN and logistic regression models. Methods such as ANN were found to fail to produce models that show which kansei words or design parameters affect overall preferences concerning product. Furthermore, these modeling approaches have problems of their own, such as overfitting.

According to Demirtas et al. [6], there are some drawbacks to the statistical methods used in the literature for modeling the relations between overall prefer- 
Ozsoydan F.B., Kandemir C.M., Demirtas E.A.: Neural-network-based...

ences and kansei words or design parameter levels. Some of these drawbacks are due to the SD scale, which was developed by Osgood et al. [28] to collect data for analysis. Products are typically evaluated on a scale of $1-5,1-7$, or 1-9 using negative-positive word pairs. Because such data are categorical in nature, using statistical methods such as linear regression is inappropriate. To overcome these drawbacks, Demirtas et al. [6] proposed a two-stage ordinal logistic regression (OLOGREG) approach. In this study, a synthesizing stage was included in the process, after spanning kansei word and product characteristics spaces based on a chosen kitchen faucet domain. This synthesizing stage consists of two substages. In the first substage, relations between kansei words and overall preference scores are studied using OLOGREG Model 1, which can be used when dependent variables are measured on an ordinal scale. The use of this model makes it possible to take interaction effects into account. The OLOGREG Model 1 obtained at this stage provides clues as to how the most preferred product styles are described by users and the image characteristics of these products. This model can also be used to confirm whether a product design will be highly preferred by customers. In the second substage, the relations between overall preferences scores and design parameter levels related to the appearance of the product are modeled using OLOGREG Models 2 and 3.

This research was inspired by the studies of Demirtas et al. [6] summarized above. The main purpose of this study is to propose an alternative approach to modeling consumer preferences that can be easily used by researchers in place of complex analytical methods. This approach is aimed at effectively and easily satisfying consumers' emotional expectations as part of today's customer-oriented product design focus.

In this study, 24 different ANN classification models were used to investigate the relations between the overall preferences and the kansei word scores of users. These 24 models were built by considering 3 different network structures (topologies), 2 different training algorithms and 2 different hidden neurons and classes. The critical factors that affect the accuracy of ANN models were evaluated through an analysis of variance (ANOVA), and the best ANN model was selected. A genetic algorithm, integrated with the best ANN model, is proposed in the third section of this study for use in determining the optimum levels of the design parameters related to product appearance. This integrated GA-ANN model performs the tasks of OLOGREG Models 2 and 3 by integrating the first and second design stages identified in a study by Demirtas et al. [6]. The fitness value of the GA is defined as a function of the predicted overall scores of users obtained using the best ANN model. The best design parameter levels for visual perceptions were determined using the GA. Lastly, product categories were classified as unfavorable or favorable, and three products were derived for each category.

The remainder of this study presents kansei engineering application steps for kitchen faucets, the development of the ANN model, the determination of the relation between kansei words and overall preference scores using ANN classification, the determination of the optimal design parameter levels using the GA and conclusions drawn from the results of the study. 


\section{Kansei engineering application steps for kitchen faucets}

In this study, the concept of kansei engineering was applied according to two definition processes, each spanning a vector space corresponding to a product domain chosen beforehand. These processes span the semantic space of kansei words and the product characteristics space. User feelings are expressed in the semantic space. After spanning the spaces, the product characteristics (design parameters and related levels) that affect user feelings are determined in the synthesizing stage. Verification studies are then carried out, and both vector spaces are updated if necessary. The synthesizing process is then repeated $[6,32]$.

As mentioned in the previous study by Demirtas et al., [6] kitchen faucets have more product characteristics than sink and bath faucets that can affect users' emotions and feelings. Therefore, kitchen faucets, specifically, standard home-type models, which are extensively produced by six leading companies in the domestic market under study, were chosen as the subject matter. These standard models are preferred by most users. They are fixed on the sink and their spouts are on their bodies. Because it is difficult and time consuming for users to evaluate many products, the study was restricted to mixer-type models (with single on-off handles) with similar prices.

After a product is chosen, kansei words are listed as negative-positive word pairs. In this study, 11 kansei word pairs were used. These word pairs are shown in Tab. I, adapted from Demirtas et al. [6]. Because users are asked to evaluate drawings of the products using these words, it is essential that words that express visual perceptions are highlighted. To assess users' overall preferences, the "overall bad-good" word pair was added to the list.

To identify product characteristics and the levels that help to define different product styles, technical documents about the product, literature related to the subject matter, manuals, and interviews with experts and experienced users were utilized, as suggested by Shütte [32].

The mixer-type kitchen faucets were divided into two groups, each with unique characteristics and levels. The groups were formed on the basis of the position of the handle (i.e., the handle being on the top or on the side). The same product characteristics and design parameter levels used in Demirtas et al. [6] were adopted in this study and are shown in Tabs. II and III.

For kitchen faucets with handles on the side, the total number of design alternatives is 216. For those with handles on the top, the total is 144 . However, it was neither practical nor necessary to develop a prototype or a picture for each of these alternative designs. Moreover, asking the users to rank or grade all possible design alternatives might not have led to reliable results. A fractional factorial experimental design similar to Taguchi's L-16 orthogonal array [30] was judged to be sufficient to explore the relations between the kansei words (or design parameters) and overall preferences. Using this experimental layout, 16 different combinations of design parameter levels were obtained for each of the two product groups (those with handles on the top and those with handles on the side). Three-dimensional (3-D) drawings (front and side views) of these product designs (styles) were created with the help of industrial product designers. Users' visual perceptions were then 
Ozsoydan F.B., Kandemir C.M., Demirtas E.A.: Neural-network-based...

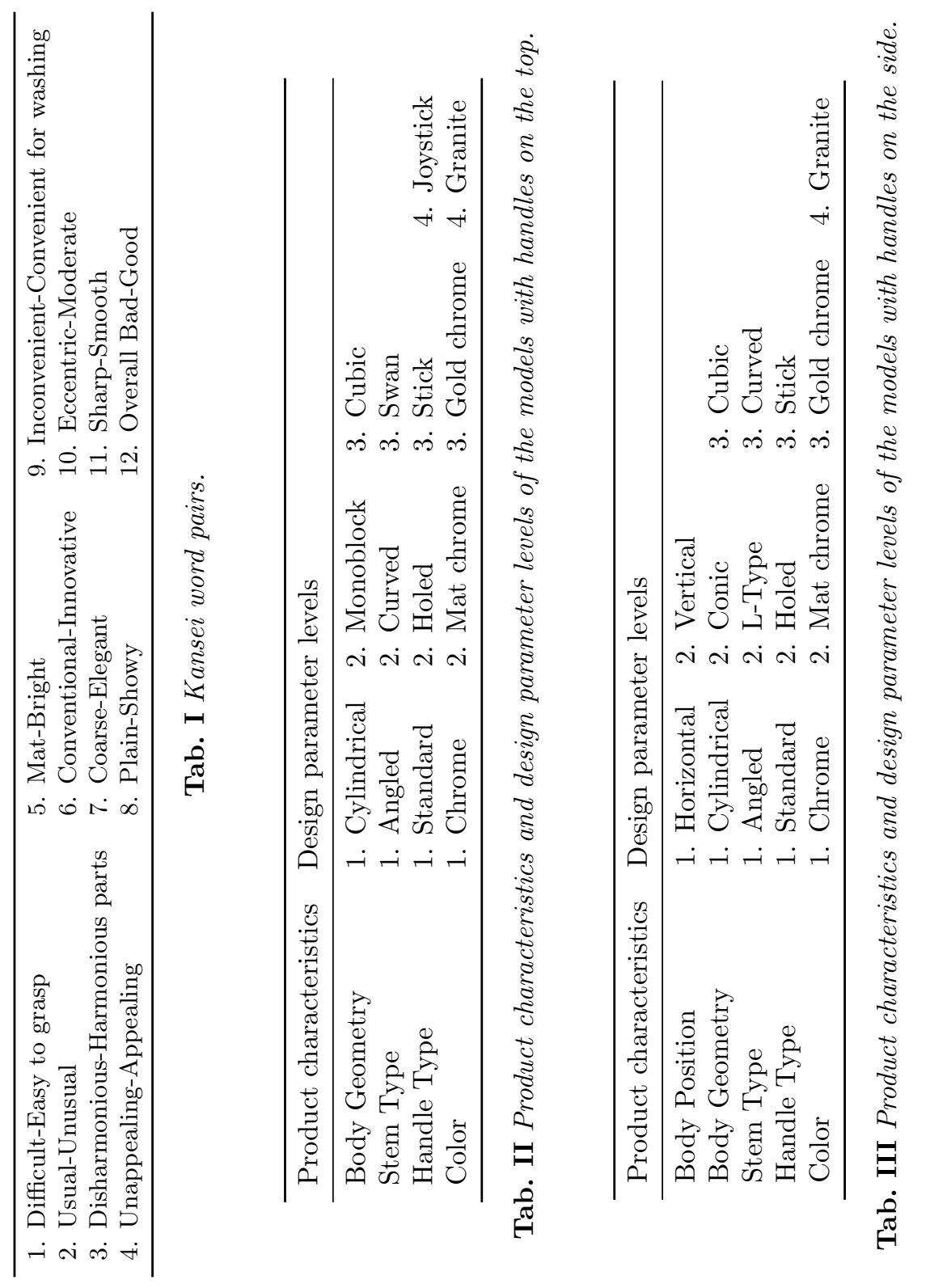


measured for each of these drawings, as well as for three product styles currently available on the market for each of the two product groups. Some of the drawings developed for models with handles on the top are shown in Fig. 1. The last three products in these figures are those which were already available on the market.

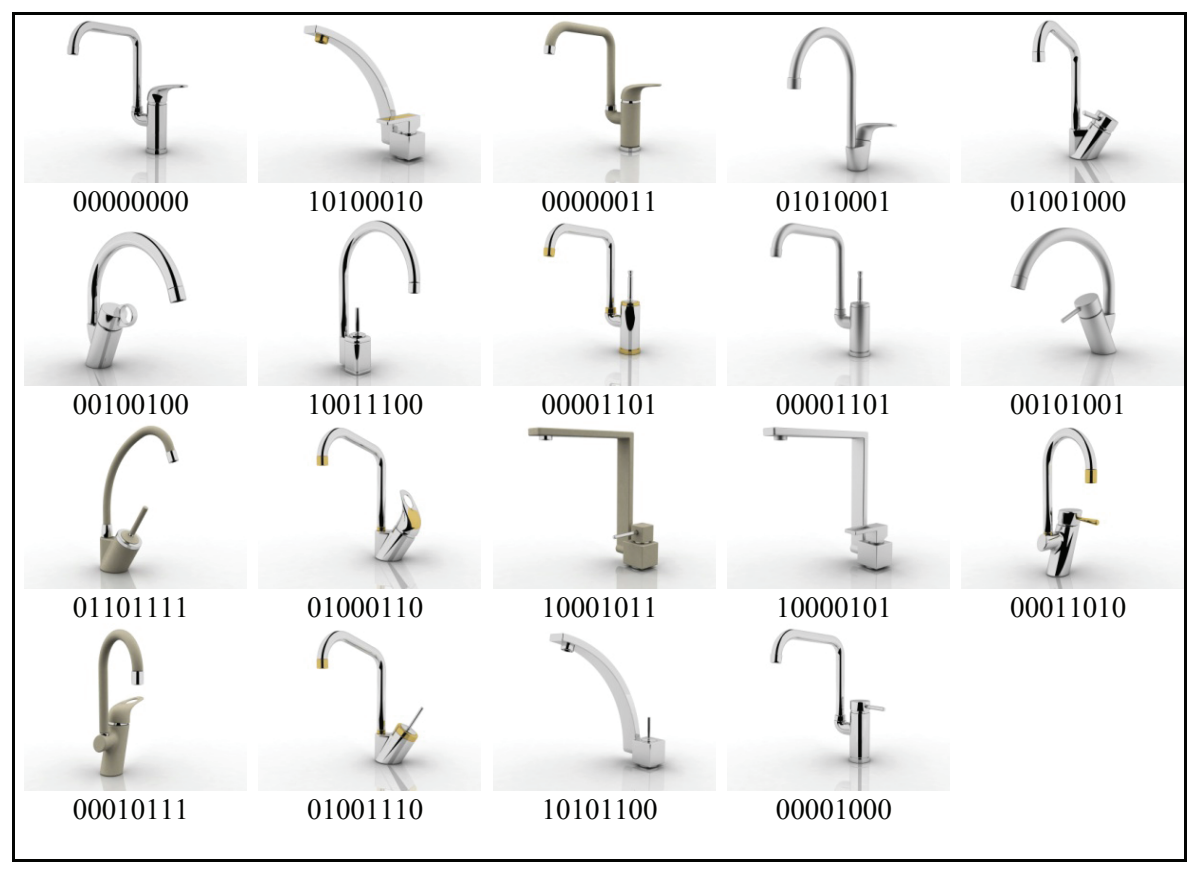

Fig. 1 Faucet styles (handle on the top).

The data necessary for the synthesizing stage were collected using a questionnaire designed by Demirtas et al. [6] for users to match kansei words with the product drawings. The questionnaire had two sections. The first section had demographic questions that asked the users for personal information concerning their gender, marital status, social status, level of income and age. In the second section, an SD scale was used to evaluate the product styles with kansei words. For each product style and kansei word pair, the user was asked to choose a number between 1 and 7, 1 corresponding to the negative word in the pair and 7 corresponding to the positive word. The reliability, factor structure and validity of the questionnaire were tested in the previous study. The total number of users who were targeted for the product groups in the domestic market was not known precisely. Therefore, the user ratios preferring $(p)$ and not preferring (1-p) a brand were assumed to be 0.5 (as suggested for such cases by [18]). The required sample size at the 0.05 significance level with 0.01 error was calculated to be 96 . Because women constituted $70 \%$ of the user population, a proportional distribution approach to stratified sampling was used, and 100 users, 70 of which were women and 30 of which were men, were administered the questionnaire. Other characteristics, such as the age, income level and marital status of the participants, were also considered in the selection of a representative sample. 


\section{ANN model development}

Dreiseitl and Machado [7] showed that logistic regression and ANN share common roots in statistical pattern recognition and that the latter can be seen as a generalization of the former. They briefly compared these two methods with other classification algorithms used in the machine learning field, such as the $\mathrm{k}$ nearest neighbors, decision trees, and support vector machines. They reviewed 72 papers that compared the classification performance of ANN and logistic regression models. For both logistic regression and ANN, the model parameters are determined by maximum likelihood estimation. Although the functional forms of logistic regression and ANN models are quite different, a network without a hidden layer is actually identical to a logistic regression model if the logistic (sigmoid) activation function is used.

In logistic regression, the model complexity is already low, especially when no or few interaction terms and variable transformations are used. Performing variable selection is a way of reducing a model's complexity and consequently decreasing the risk of overfitting. As mentioned before, overfitting may cause a loss in the model's flexibility. Compared to logistic regression, ANN models are more flexible and thus more susceptible to overfitting.

ANN models have good learning abilities and for this reason have been successfully applied in various research areas [27, 38]. As mentioned before, 24 ANN models were developed to examine the relation between kansei words and overall preference scores, using various numbers of network architectures, training algorithms, hidden layers and classes.

Feedforward neural networks consist of a series of layers. The first layer has a connection to the network input. Each subsequent layer has a connection to the previous layer. The final layer produces the network's output. Feedforward networks can be used for any type of input-output mapping. The information flow between the network layers is unidirectional. A feedforward network with one hidden layer and enough neurons in the hidden layers can fit any finite inputoutput mapping problem. Fitting networks are feedforward neural networks used to fit an input-output relationship. Pattern recognition networks are feedforward networks that can be trained to classify inputs according to target classes. The target data for pattern recognition networks should consist of vectors of all zero values except for a value of 1 for element $i$, where $i$ is the class to be represented $[1]$.

After the creation of the network architecture, the next step is to apply supervised learning to train a neural network to learn the different decision regions for the given input data. Supervised learning of neural networks can be considered as a function optimization problem in which higher-order optimization methods using gradient information are used to improve the rate of convergence [35]. In this study, the performance of the Levenberg-Marquardt (LM) algorithm [1] and the scale conjugate gradient (SCG) algorithm [23] were studied. Both of the algorithms have been widely applied to the function approximation problem. Because of the use of a second derivative to update the weights, the LM algorithm yields lower mean square errors than any of the other algorithms tested. However, when the input data set is large, the calculation becomes a time-consuming process. The SCG 
algorithm is almost as fast as the LM algorithm in solving function approximation problems and requires fewer calculations than the LM algorithm, but it is not as precise as the LM algorithm. The trade-off is that the SCG algorithm can be used with large sets of data and has only modest memory requirements [9].

In this study, ANN models were developed using two of the four most commonly used calculations for determining the number of neurons in a single hidden layer [22], listed below as formulations 1 and 2:

$$
\begin{aligned}
& \sqrt{\text { the number of input neurons }+ \text { the number of output neurons }} \\
& \frac{\text { the number of input neurons }+ \text { the number of output neurons }}{2}
\end{aligned}
$$

The ANN structures used in this study are shown in Fig. 2.

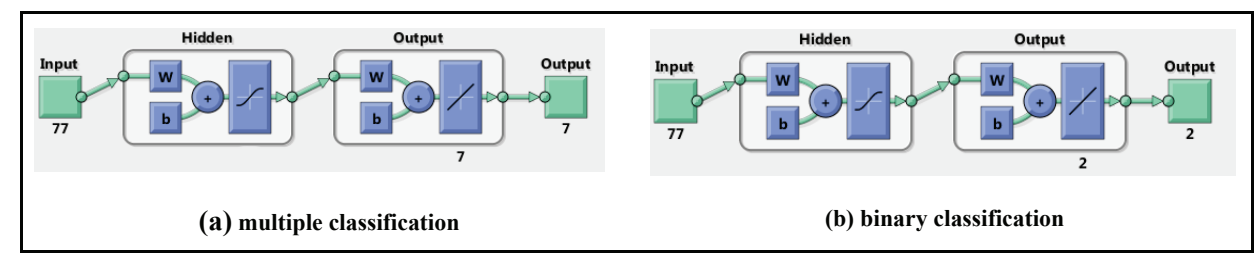

Fig. 2 Artificial Neural Network structure.

Data transformation All of the kansei word scores and overall preferences were transformed into appropriate input and output vectors for training purposes. Two types of transformations were used: mapping-7 for multiple classification purposes ( 7 classes) and mapping-2 for binary classification purposes (2 classes).

$S$ : Kansei score, ranging from 1 to 7 .

$O$ : Overall preference, ranging from 1 and 7 .

Mapping-7 transformation:

$$
\begin{array}{cc}
X=\left[x_{1}, x_{2}, \ldots, x_{7}\right]^{\mathrm{T}}: & \text { transformation vector of } S \\
\vdots & \\
Y=\left[y_{1}, y_{2}, \ldots, y_{7}\right]^{\mathrm{T}}: & \text { transformation vector of } O \\
\vdots & \\
x_{i}=1 \text { for } S=i \text { and } x_{i}=0 \text { for } S \neq i & \\
y_{i}=1 \text { for } O=i \text { and } y_{i}=0 \text { for } O \neq i &
\end{array}
$$


Ozsoydan F.B., Kandemir C.M., Demirtas E.A.: Neural-network-based...

Mapping-2 transformation:

$$
\begin{array}{cc}
Y=\left[y_{1}, y_{2}\right]^{\mathrm{T}}: & \text { transformation vector of } O \\
\vdots & \\
y_{1}=1 \text { for } O<6, \text { otherwise } y_{1}=0 & \\
y_{2}=1 \text { for } O \geq 6, \text { otherwise } y_{2}=0 &
\end{array}
$$

\section{Determination of the relation between Kansei words and overall preference scores using the ANN model}

To investigate the relations between the overall preferences and kansei word scores of the users, 24 ANN models were developed, considering critical factors such as the training algorithm, the structure, the number of hidden layers and the number of classes. These factors are summarized for the 24 models in Tab. IV. To determine

\begin{tabular}{llcccc}
\hline Model & $\begin{array}{l}\text { Network } \\
\text { architecture }\end{array}$ & Algorithm & $\begin{array}{c}\text { Hidden } \\
\text { layers }\end{array}$ & $\begin{array}{c}\text { Class } \\
\text { number }\end{array}$ & $\begin{array}{c}\text { Accuracy } \\
{[\%]}\end{array}$ \\
\hline 1 & fitnet & LM & 1 & 7 & 48.7 \\
2 & fitnet & LM & 1 & 2 & 90.0 \\
3 & fitnet & LM & 2 & 7 & 85.2 \\
4 & fitnet & LM & 2 & 2 & 100.0 \\
5 & fitnet & SCG & 1 & 7 & 36.8 \\
6 & fitnet & SCG & 1 & 2 & 94.8 \\
7 & fitnet & SCG & 2 & 7 & 73.6 \\
8 & fitnet & SCG & 2 & 2 & 99.3 \\
9 & fforward & LM & 1 & 7 & 47.4 \\
10 & fforward & LM & 1 & 2 & 95.4 \\
11 & fforward & LM & 2 & 7 & 80.3 \\
12 & fforward & LM & 2 & 2 & 80.6 \\
13 & fforward & SCG & 1 & 7 & 38.6 \\
14 & fforward & SCG & 1 & 2 & 93.5 \\
15 & fforward & SCG & 2 & 7 & 55.4 \\
16 & fforward & SCG & 2 & 2 & 99.6 \\
17 & pnet & LM & 1 & 7 & 45.2 \\
18 & pnet & LM & 1 & 2 & 98.6 \\
19 & pnet & LM & 2 & 7 & 78.4 \\
20 & pnet & LM & 2 & 2 & 100.0 \\
21 & pnet & SCG & 1 & 7 & 41.1 \\
22 & pnet & SCG & 1 & 2 & 96.5 \\
23 & pnet & SCG & 2 & 7 & 79.0 \\
24 & pnet & SCG & 2 & 2 & 98.8 \\
\hline & & & & & \\
\hline
\end{tabular}

Tab. IV The accuracy [\%] of the ANN models. 
the critical factors that affect the accuracy of a model and to find the best factor levels for multiple classifications, an analysis of variance (ANOVA) was conducted. The results are presented in Tab. V, where DF, Seq SS, Adj SS, Adj MS, F, $\mathrm{P}$ and $\mathrm{S}$ represent the degrees of freedom, sequential sums of squares, adjusted sums of squares, adjusted mean squares, $\mathrm{F}$ and $\mathrm{P}$ values and standard deviance, respectively.

\begin{tabular}{lcrrrrr}
\hline \multicolumn{1}{l}{$\begin{array}{l}\text { Analysis Of Variance for Accuracy using Adjusted SS for Tests } \\
\text { Source }\end{array}$} & DF & Seq SS & Adj SS & Adj MS & F & P \\
\hline Topology & 2 & 82.93 & 82.93 & 41.46 & 1.38 & 0.420 \\
Algorithm & 1 & 307.04 & 307.04 & 307.04 & 10.22 & 0.085 \\
HL & 1 & 3139.57 & 3139.57 & 3139.57 & 104.51 & 0.009 \\
Topology*Algorithm & 2 & 118.01 & 118.01 & 59.00 & 1.96 & 0.337 \\
Topology*HL & 2 & 84.98 & 84.98 & 42.49 & 1.41 & 0.414 \\
Algorithm*HL & 1 & 10.27 & 10.27 & 10.27 & 0.34 & 0.618 \\
Error & 2 & 60.08 & 60.08 & 30.04 & & \\
Total & 11 & 3802.87 & & & & \\
& & & & & & \\
S $=5.480$ & & & & & & \\
88 R-Sq = 98.42\% R-Sq(adj) $=91.31 \%$ & &
\end{tabular}

Tab. V ANOVA results for multiple classifications.

The ANOVA results shown in Tab. V indicate that the training algorithm and the number of hidden layers are critical factors that affect the accuracy at the 0.10 significance level $(p<0.10)$. The topology (structure) and the interaction effects of the topology, the training algorithm and the number of hidden layers are not critical factors. The plots of main effects shown in Fig. 3 indicate that the LM algorithm with two hidden layers yields the highest accuracy.

When binary classification is used (favorable is indicated by a score of 6 or more; unfavorable is indicated by a score less than 6), the accuracy of all the ANN models is greater than or equal to $90 \%$. For some ANN models, the accuracy is rather good for multiple classifications. For instance, the accuracy of Model 3 (the LM algorithm with two hidden layers and a fitnet structure) is $85.2 \%$ for multiple classifications and $100 \%$ for binary classification.

\section{Determination of the optimal design parameter levels with GA}

The concept of a genetic algorithm was first proposed by John Holland in the early 1970s. A genetic algorithm simulates natural evolution processes, such as crossover, mutation and natural selection that favor the survival of organisms [11]. GA is a very efficient population-based search method that has been applied to many optimization and classification problems $[4,8]$. 
Ozsoydan F.B., Kandemir C.M., Demirtas E.A.: Neural-network-based...

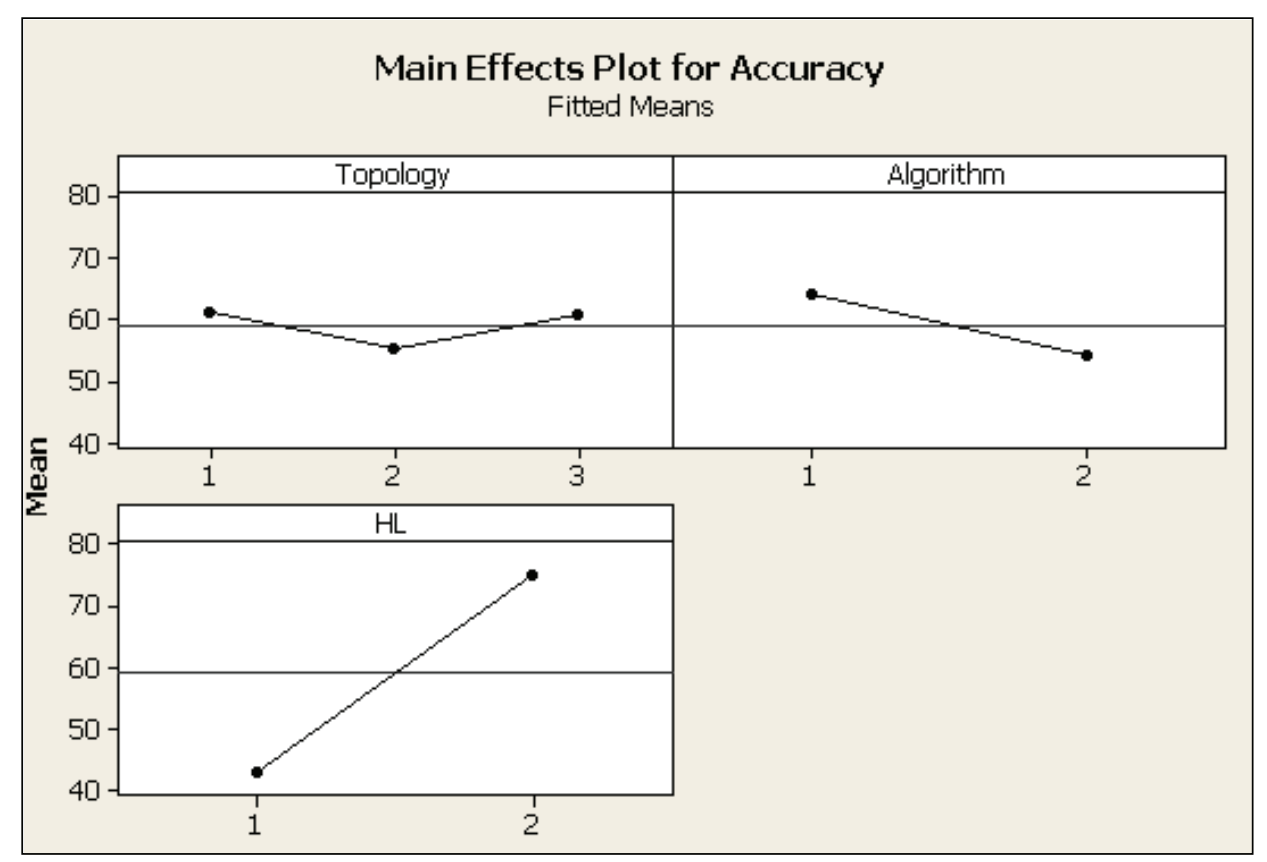

Fig. 3 Main effect plots for accuracy.

For the proposed GA, a fitness value was defined as the sum of the predicted overall preferences scores of the users. The predictions were performed using the ANN model identified as being the best (ANN Model 3), based on the statistical analysis results. The goal of the GA is to find the best design parameter levels for visual perceptions by maximizing the overall preference scores. Additionally, according to the GA results, product categories were classified as being unfavorable or favorable, and for each category, 3 products were categorized. The general framework of the GA (Fig. 4) used in this study and the required information are summarized below.

- In general, a larger population might be more effective but require more calculation time. Hence, the population size was fixed at 10.

- Crossover and mutation operations were performed according to crossover and mutation rates of 0.15 and 0.05 , respectively.

- The GA interacts with the ANN model and uses the predicted overall preference scores derived from ANN Model 3 as fitness values.

- If necessary (when a better solution is found), incumbent solutions for each product group are updated.

- Two features were considered as termination criteria in this study. The first criterion was a limit on the number of iterations, and the second criterion was the attainment of probabilities greater than 0.95 for three products in 
each product group. If at least one of these two conditions was satisfied, the algorithm was terminated.

- With a tournament size of 2, a tournament selection was performed and a new generation was created.

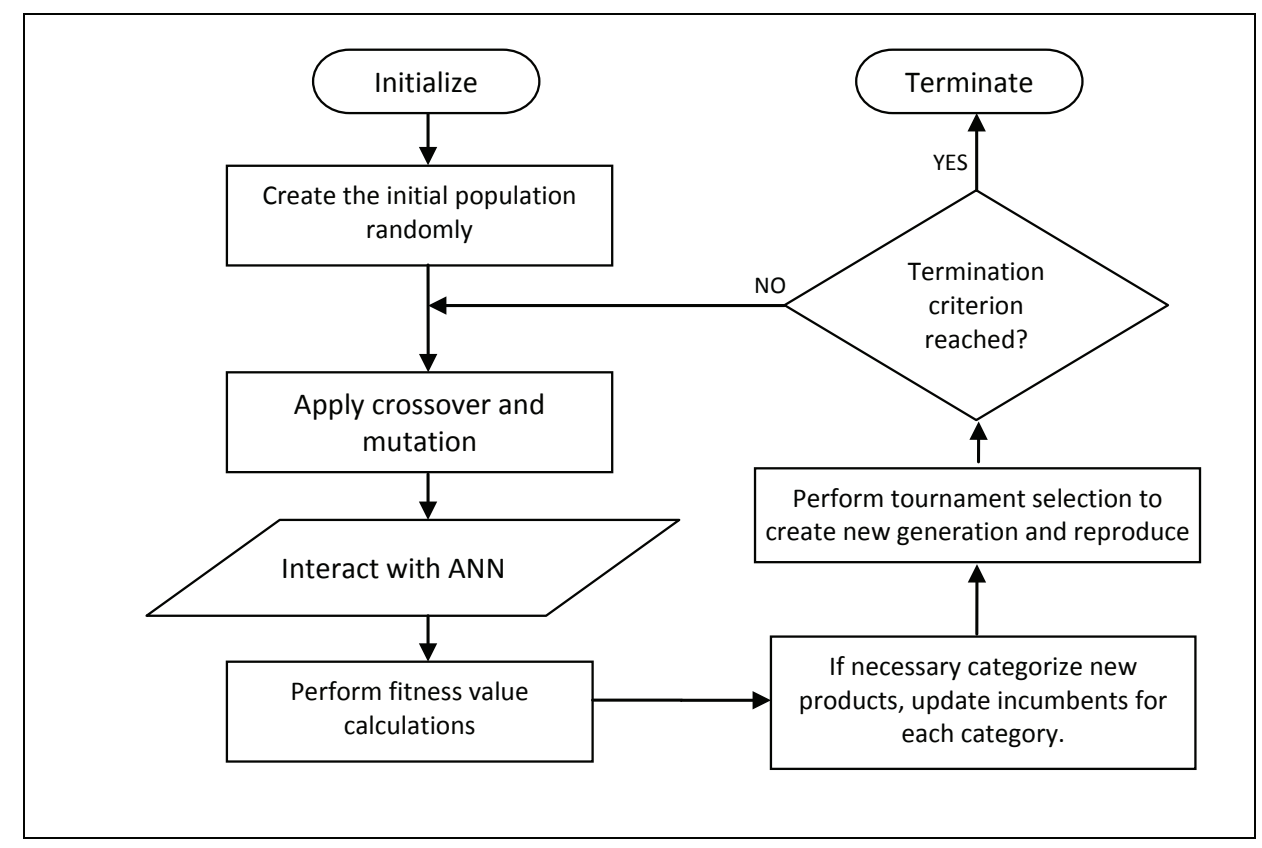

Fig. 4 Flow diagram of the GA.

To show combinations of kitchen faucet features, binary representation encoding was used. For the kitchen faucet models with handles on the top, as shown in Tab. II, there are 4 factors, with 3, 3, 4, and 4 design parameter levels. For each factor, 2 bits are used to encode a model with a handle on the top. For instance, a cylindrical body geometry, a curved stem type, a stick handle type and a granite color for a kitchen faucet model with a handle on the top is encoded as the chromosome shown in Fig. 5(a).

The factors and design parameter levels for the kitchen faucet models with handles on the side are given in Tab. III. There are 5 factors for this model. Therefore, one more bit is required to encode a 2-level factor. For example, the solution representation for a kitchen faucet with a horizontal body position, a cubic body geometry, an L-type stem, a standard handle type, a mat chrome color and a handle on the side is presented in Fig. 5(b). The genetic codes (encoded representations) for 19 kitchen faucet models with handles on the top are shown in Fig. 1.

For a better understanding, the framework of the proposed integrated approach (integrating analytic and artificial intelligence techniques) is finally obtained as given in Fig. 6. As one can see from this figure, analytic approaches constitute the 


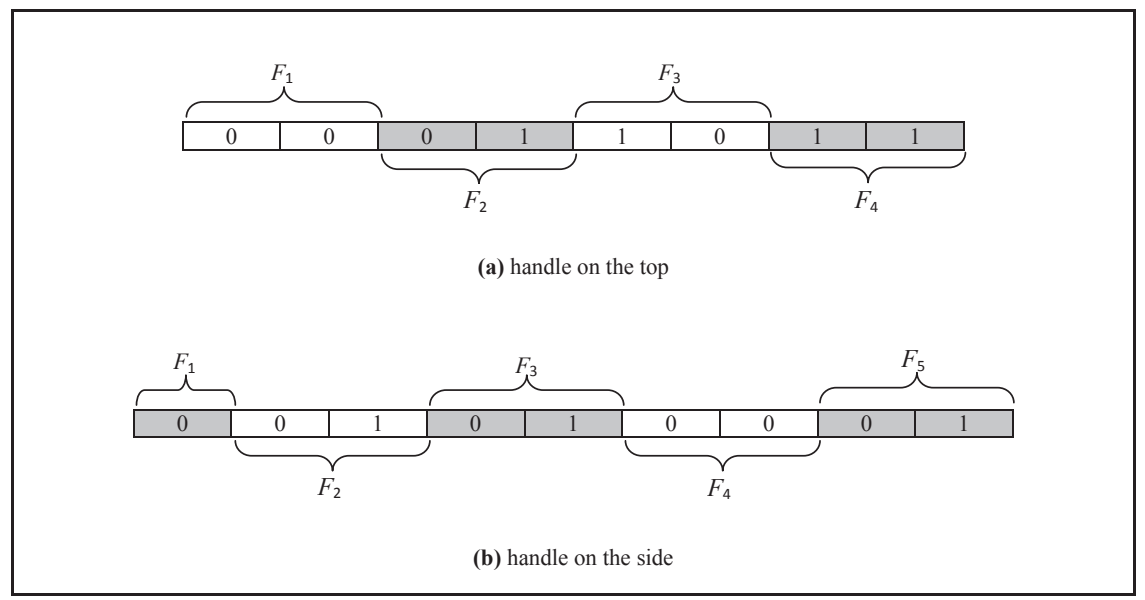

Fig. 5 An encoded solution representation.

initial stages of the proposed approach, where artificial intelligence techniques use the data gathered by those techniques.

The developed approach in this study was applied separately to kitchen faucets with handles on the side and with handles on the top, to identify favorable and unfavorable product categories for each of these two types of models. The first category (unfavorable) includes the products for which the predicted overall scores were less than or equal to 3 , whereas the second category (favorable) includes products with overall scores greater than or equal to 6 .

The design parameter levels of the most favorable and the most unfavorable products for the different types of kitchen faucet styles (with handles on the side and handles on the top) are presented in Tabs. VI and VII, along with the corresponding GA fitness values. The most favorable and unfavorable kitchen faucet styles are illustrated in Figs. 7(a) and (b).

The number of factors and levels considered in this study were reasonable in comparison with those used in other related studies. However, the significant contribution of GA, versus statistical techniques such as Hayashi's quantification theory, linear regression, conjoint analysis and OLOGREG, is the ability to consider many factors and levels in the determination of optimal design parameter levels.

The results obtained using the proposed approach are similar to those previously reported by Demirtas et al. [6]. The results of this study show that one of the widely used evolutionary algorithms, GA, is an effective approach in determining optimal design parameters. However, the success of GA in this application depends on the adequacy of the preliminary work, such as the questionnaire study, the experimental design, the generation of realistic 3D drawings of new products, the creation of effective ANN models and the ANOVA. Therefore, GA cannot be considered a stand-alone approach but rather an approach that is effective when integrated with other tools, as shown in this study. As a result, it was demonstrated in the present study that, the integrated GA-ANN model approach used in this study can be an effective alternative to complex and challenging analytic methods. 


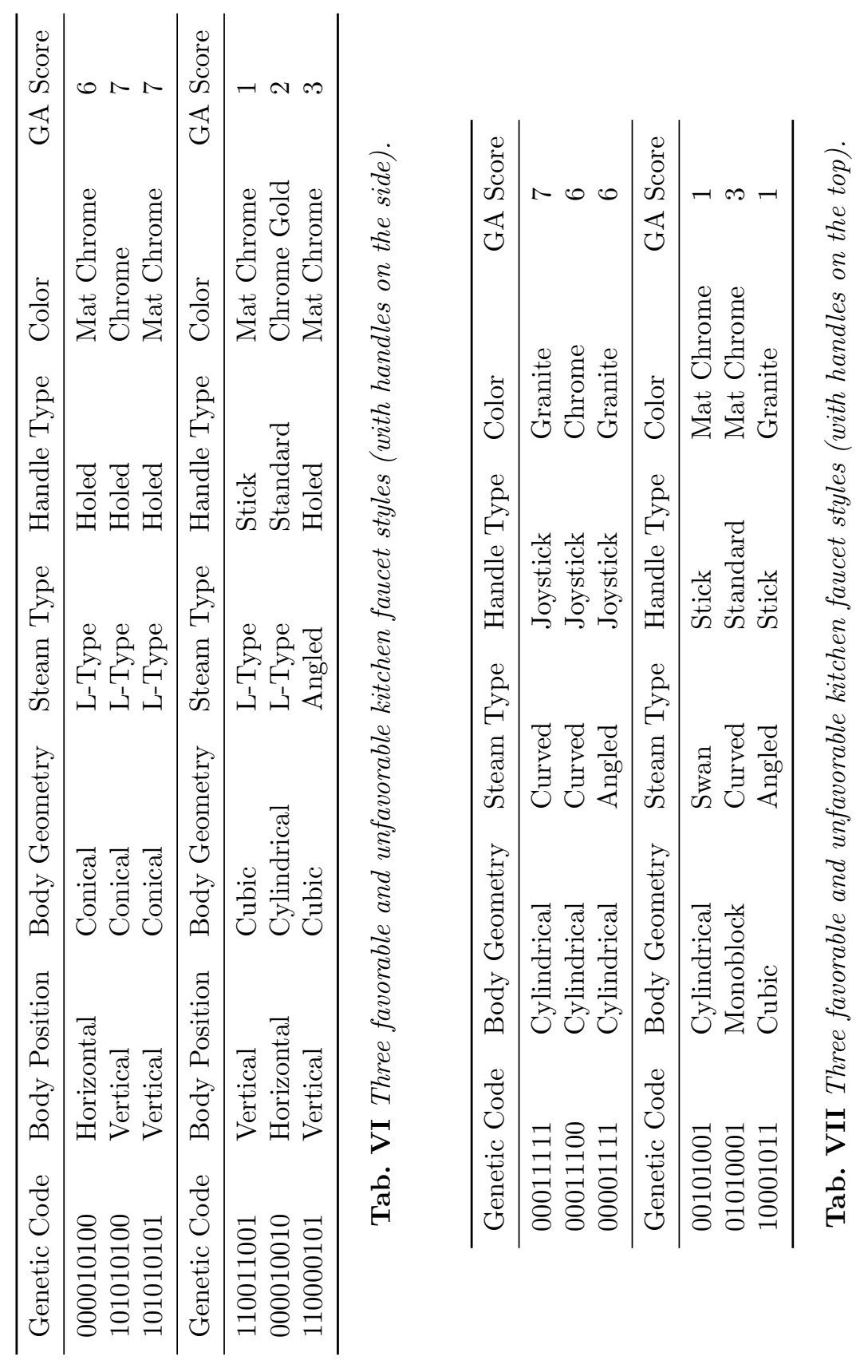


Ozsoydan F.B., Kandemir C.M., Demirtas E.A.: Neural-network-based...

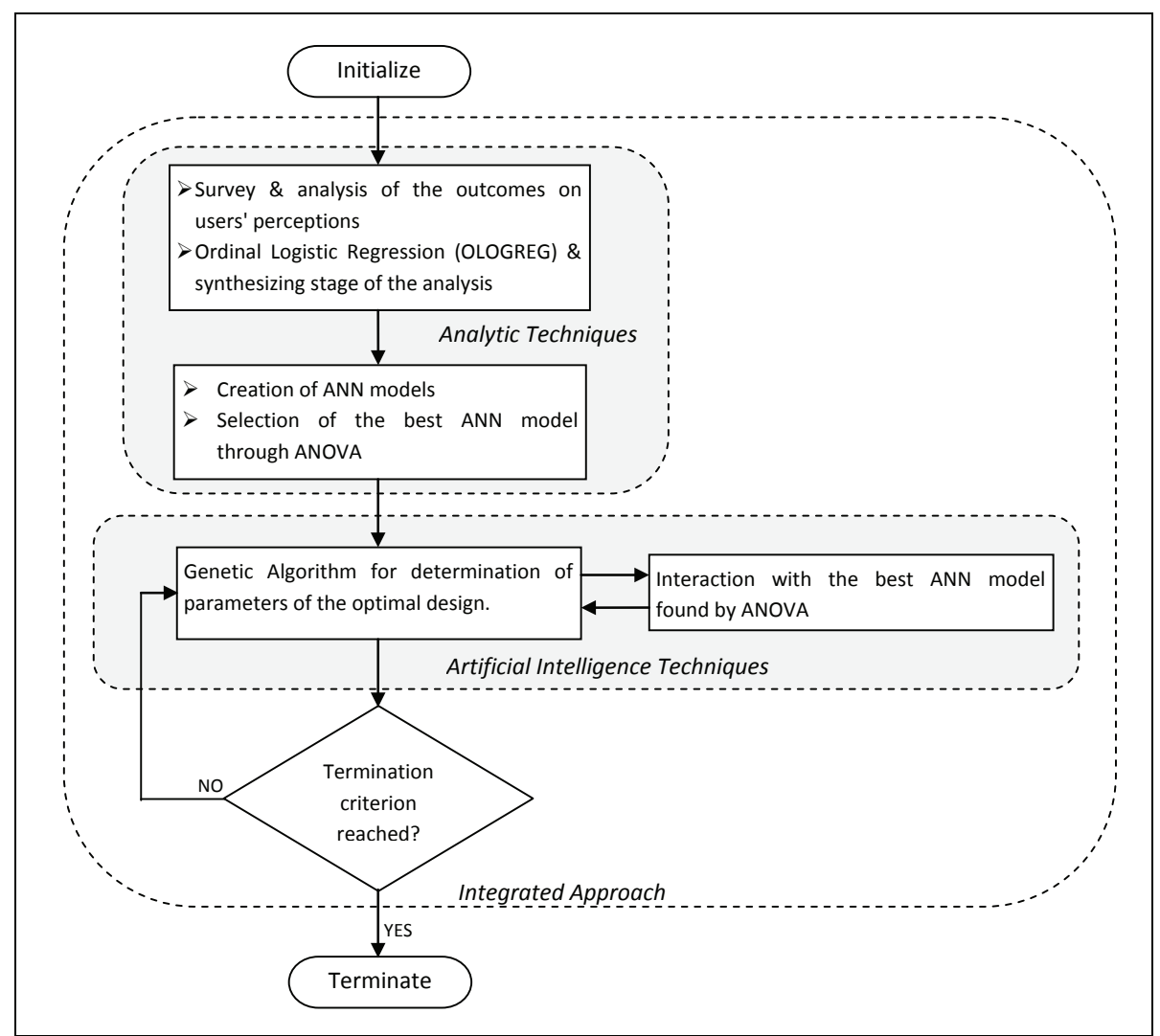

Fig. 6 A flow chart of the proposed integrated approach.

\section{Conclusion}

This study presents an integrated approach to determining the best design parameters for the design of industrial products by considering the perceptional dimension of quality. The use of artificial intelligence techniques is discussed, and combining techniques to overcome limitations encountered in previous studies is recommended.

In applications of this type of integrated approach, the following points warrant particular attention: the selection of appropriate customer (user) segments; a thorough understanding of customer needs and expectations; the determination of kansei word pairs in the native language of the customers; the selection of a representative group of (potential or real) customers for questionnaire applications; the selection of design parameter levels that are not influenced prematurely by cost and technology limitations; the determination of product style alternatives using fractional factorial designs; pre-processing of the collected data to identify missing values, inconsistencies and correlations; and appropriate interpretation and confirmation of the results.

The product styles determined in this manner should be developed into detailed designs for which cost, technology, performance, reliability, durability and safety 


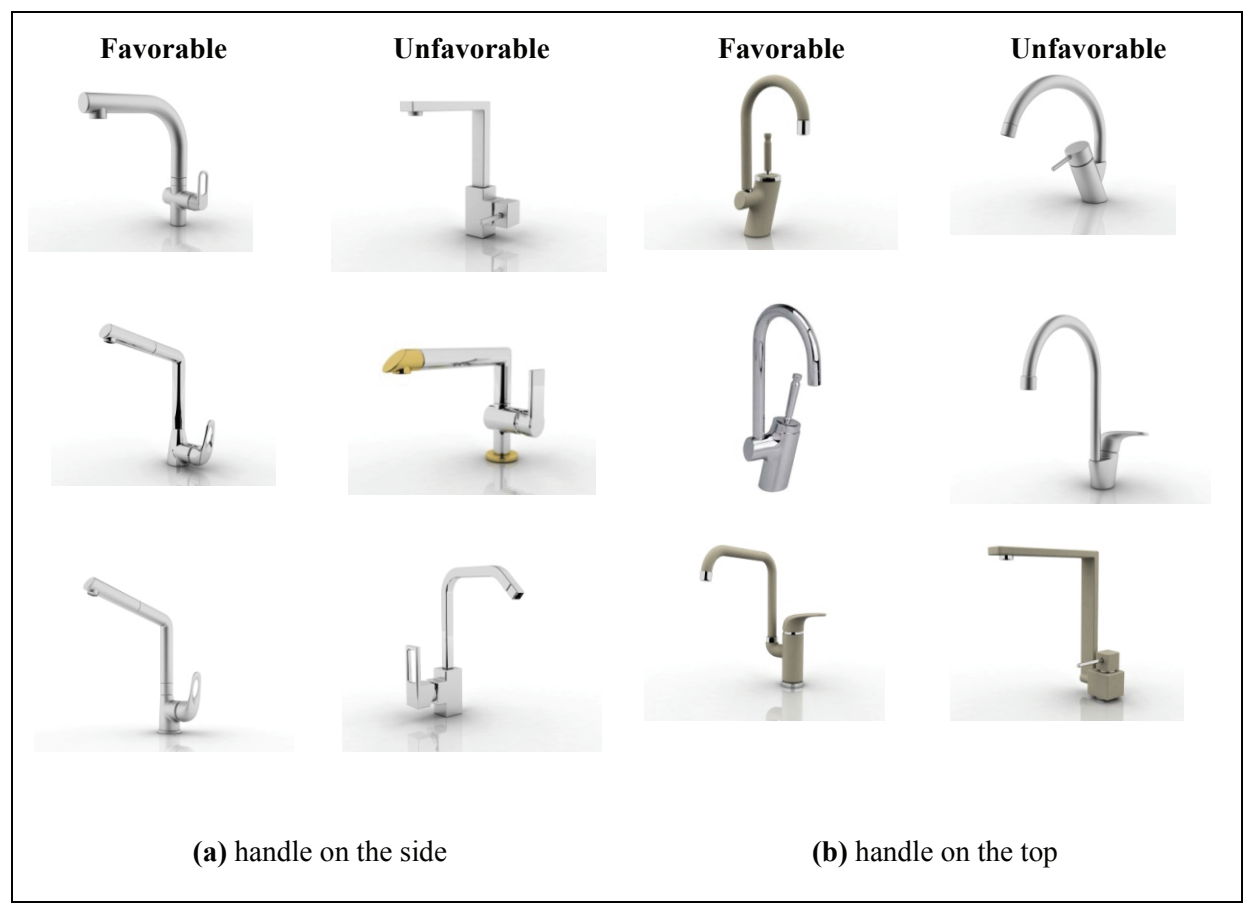

Fig. 7 Kitchen faucet styles identified using the GA.

are considered. Innovative solutions that preserve the recommended styles but yield high performance at low cost should be sought. In any case, style change requests found to be necessary at other product development stages can be evaluated, and visual perceptions of them being of sufficiently high quality can be confirmed using the models developed according to the approach proposed in this study.

This study proposes a hybrid approach to ANN and GA, although it considers fewer design attributes and levels than other studies described in the literature. This approach was applied to two types of kitchen faucet styles. The modeling and optimization approach developed and demonstrated in this study can be adapted to any product design and is particularly well suited to the consideration of product styles and the visual appearance of products.

As the results of this study show, the proposed integrated approach is an effective method for revealing user perceptions in new product development. Therefore, the proposed approach can be used in product design problems as an effective alternative to several complex analytical methods to assess consumers' emotional preferences in product development and design.

Although it requires specialized package programs, interactively creation of 3D models of industrial products can be taken into consideration as a future research. Thus, some stages of the proposed integrated approach might be eliminated. 
Ozsoydan F.B., Kandemir C.M., Demirtas E.A.: Neural-network-based...

\section{References}

[1] BEAlE M.H., HAGAN M.T., DEMUTh H.B. Neural Network Toolbox 7 User's Guide. 2010, The Math Works Inc., Natick, MA. Available from: http://www . mathworks. com/help/pdf_doc/nnet/nnet_ug.pdf

[2] BLIJLEVENS J., CREUSEN M.E.H., SCHOORMANS J.P.L. How consumers perceive product appearance: the identification of three product appearance attributes. Int. J. Des. 2009, 3(3), pp. 27-35. Available from: http://www.ijdesign.org/ojs/index.php/IJDesign/article/view/535

[3] BLOCH P.H. Seeking the ideal form: product design and consumer response. J. Marketing. 1995, 59(3), pp. 16-29, doi: 10.2307/1252116.

[4] CHAMBER L. The Practical Handbook of Genetic Algorithms. Boca Raton, FL: CRC Press, 1995, doi: 10.1201/9781420050073.

[5] CHANG H.C., LAI H.H., CHANG Y.M. Expression modes used by consumers in conveying desire for product form: a case study of a car. Int. J. Ind. Ergonom. 2006, 36, pp. 3-10, doi: 10.1016/j.ergon.2005.06.004.

[6] DEMIRTAS E.A., ANAGUN A.S., KOKSAL G. Determination of optimal product styles by ordinal logistic regression versus conjoint analysis for kitchen faucets. Int. J. Ind. Ergonom. 2009, 39, pp. 866-875, doi: 10.1016/j.ergon.2009.06.007.

[7] DREISEITL S., OHNO-MACHADO L. Logistic regression and artificial neural network classification models: a methodology review. J. Biomed. Inform. 2002, 35, pp. 352-359, doi: 0.1016/S1532-0464(03)00034-0.

[8] GOLDBERG D.E. Genetic Algorithms in Search, Optimization and Machine Learning. Reading, MA: Addison-Wesley, 1989, doi: 10.5860/choice.27-0936.

[9] GRAY D., ZHANG Z., APOSTOAIA C., XU C. A neural network based approach for the detection of faults in the brushless excitation of a synchronous motor. In: Proceedings of the 9th IEEE International Conference on Electro/Information Technology, Windsor, Ontario. IEEE, 2009, pp. 423-428, doi: 10.1109/EIT.2009.5189654.

[10] HARTONO M., CHUAN K. How the Kano model contributes to kansei engineering in services. Ergonomics. 2011, 54(11), pp. 987-1004, doi: 10.1080/00140139.2011.616229.

[11] HOLLAND J.H. Adaptation in Natural and Artificial Systems. 2nd ed. MIT press, 1992.

[12] HSIAO S.W., CHIU F.Y., LU S.H. Product-form design model based on genetic algorithms. Int. J. Ind. Ergonom. 2010, 40, pp. 237-246, doi: 10.1016/j.ergon.2010.01.009.

[13] HSIAO S.W., HUANG H.C. A neural network based approach for product form design. Design Stud. 2002, 23, pp. 67-84, doi: 10.1016/S0142-694X(01)00015-1.

[14] HSIAO S.W., TSAI H.C. Applying a hybrid approach based on fuzzy neural network and genetic algorithm to product form design. Int. J. Ind. Ergonom. 2005, 35(5), pp. 411-428, doi: 10.1016/j.ergon.2004.10.007.

[15] HSU S.H., CHUANG M.C., CHANG C.C. A semantic differential study of designers' and users' product form perception. Int. J. Ind. Ergonom. 2000, 25(4), pp. 375-391, doi: 10.1016/S0169-8141(99)00026-8.

[16] KELLY J. Interactive genetic algorithms for shape preference assessment in engineering design [online]. Ann Arbor MI, 2008. Ph.D. thesis, University of Michigan [viewed 2014-1126]. Available from: http://deepblue.lib.umich.edu/handle/2027.42/60812

[17] KIM H.S., CHO S.B. Application of interactive genetic algorithm to fashion design. Eng. Appl. Artif. Intel. 2000, 13, pp. 635-644, doi: 10.1016/S0952-1976(00)00045-2.

[18] KISH L. Survey Sampling. New York: John Wiley and Sons Inc, 1995.

[19] LAI H.H., CHANG Y.M., CHANG H.C. A robust design approach for enhancing the feeling quality of a product: a car profile case study. Int. J. Ind. Ergonom. 2005, 35(5), pp. 445-460, doi: $10.1016 /$ j.ergon.2004.10.008.

[20] LAI H.H., LIN Y.C., YEH C.H. Form design of product image using grey relational analysis and neural network models. Comput. Oper. Res. 2005, 32, pp. 2689-2711, doi: 10.1016/j.cor.2004.03.021. 
[21] LAI H.H., LIN Y.C., YEH C.H., WEI C.H. User-oriented design for the optimal combination on product design. Int. J. Prod. Econ. 2006, 100(2), pp. 253-267, doi: 10.1016/j.ijpe.2004.11.005.

[22] LIN Y.C., YEH C.H., HUNG C.H. A neural network approach to the optimal combination of product color design. In: Proceedings of the Fourth International Conference on Networked Computing and Advanced Information Management, Gyeongju, South Korea. IEEE, 2008, pp. 53-57, doi: 10.1109/NCM.2008.194.

[23] MOLLER M.F. A scaled conjugate gradient algorithm for fast supervised learning. Neural Networks. 1993, 6, pp. 523-533, doi: 10.1016/S0893-6080(05)80056-5.

[24] MOSHAGEN M., MUSCH J., GÖRITZ A.S. A blessing, not a curse: experimental evidence for beneficial effects of visual aesthetics on performance. Ergonomics. 2009, 52(10), pp. 13111320, doi: 10.1080/00140130903061717.

[25] NAGAMACHI M. Kansei engineering: a new ergonomic consumer-oriented technology for product development. Int. J. Ind. Ergonom. 1995, 15, pp. 3-11, doi: 10.1016/0169-8141(95)90025-X.

[26] NAGAMACHI M. Kansei engineering as a powerful consumer-oriented technology for product development. Appl. Ergon. 2002, 33(3), pp. 289-294, doi: 10.1016/S0003-6870(02)00019-4.

[27] NELSON M. A Practical Guide to Neural Nets. New York: Addison-Wesley, 1991.

[28] OSGOOD C.E., SUCI G.J., TANNENBAUM P.H. The Measurement of Meaning. USA: University of Ilinois Press, 1957.

[29] PALIWAL M., KUMAR U. Neural networks and statistical techniques: a review of applications. Expert Syst. Appl. 2009, 36, pp. 2-17, doi: 10.1016/j.eswa.2007.10.005.

[30] PHADKE M. Quality Engineering Using Robust Design. Prentice Hall International Inc, 1989, doi: 10.1007/978-1-4684-1472-1_3.

[31] POIRSON E., PHILIPPE D., PETIOT J.F. User-centered design by genetic algorithms: application to brass musical instrument optimization. Eng. Appl. Art. Intel. 2007, 20, pp. 511-518, doi: 10.1016/j.engappai.2006.09.002.

[32] SHÜTTE S. Integrating Kansei Engineering Methodology in Product Development. Sweden: Linköping Studies in Science and Technology, 2002.

[33] SONDEREGGER A., ZBINDEN G., UEBELBACHER A., SAUER, J. The influence of product aesthetics and usability over the course of time: a longitudinal field experiment. Ergonomics. 2012, 55(7), pp. 713-730, doi: 10.1080/00140139.2012.672658.

[34] TOVEY M. Styling and design: intuition and analysis in industrial design. Design Stud. 1997, 18(1), pp. 5-31, doi: 10.1016/S0142-694X(96)00006-3.

[35] TRAN C., ABRAHAM A., JAIN L. Decision support systems using hybrid neurocomputing. Neurocomputing. 2004, 61, pp. 85-97, doi: 10.1016/j.neucom.2004.03.006.

[36] WELLINGS T., PITTS M.J., WILLIAMS M.A. Characterising the experience of interaction: an evaluation of automotive rotary dials. Ergonomics. 2012, 55(11), pp. 1298-1315, doi: 10.1080/00140139.2012.708057.

[37] YAmAmoto M., LAMBERT, D. The impact of product aesthetics on the evaluation of industrial products. J. Prod. Innovat. Manag. 1994, 11(4), pp. 309-324, doi: 10.1016/0737-6782(94)90086-8.

[38] YEH C.H., LIN Y.C. Neural network models for transforming consumer perception into product form design. Lect. Notes. Comput. Sci. 2006, 3973, pp. 799-804, doi: 10.1007/11760191_117. 